\title{
Lung and blood mononuclear cell responses of tuberculosis patients to mycobacterial proteins
}

\author{
S.B. Sable*, , D. Goyal" , I. Verma*, D. Behera" and G.K. Khuller*
}

ABSTRACT: The differences in specificity of human lung and peripheral lymphocytes for mycobacterial antigens $(\mathrm{Ag})$ need to be evaluated in order to identify vaccine candidates against pulmonary tuberculosis (TB).

Therefore, the present study examined the response to low molecular weight secretory proteins of Mycobacterium tuberculosis in bronchoalveolar lavage (BAL) and peripheral blood mononuclear cells (PBMCs) from minimal pulmonary TB and non-TB patients.

Ag85A, Ag85B, culture filtrate protein (CFP)-31, CFP-22.5, CFP-21, M. tuberculosis protein-64 and an as yet uncharacterised $19 \mathrm{kDa}$ protein were found to be predominantly recognised by BAL cells of TB patients on the basis of lymphocyte proliferation and significant interferon- $\gamma$ release. However, recognition of CFP-8, 6-kDa early secreted antigenic target, CFP-10, CFP-14.5, M. tuberculosis secretory protein-17 and five other as yet uncharacterised low molecular weight polypeptides was found to be high on the basis of lymphocyte proliferation at the level of PBMCs. Furthermore, BAL macrophages, and not blood monocytes, were found to produce nitric oxide (NO) in response to mycobacterial Ags. Among polypeptides predominantly recognised by BAL lymphocytes, only Ag85A and Ag85B were found to induce both NO and interleukin-12 (p40) by alveolar macrophages.

In conclusion, the present results indicate heterogeneity in antigen recognition by bronchoalveolar lavage cells and peripheral mononuclear blood cells of minimal tuberculosis patients, and also suggest the utility of antigen 85 complex polypeptides for the development of a future mucosal antituberculous vaccine.

KEYWORDS: Immune response, low molecular weight polypeptides, lung, peripheral blood, tuberculosis, vaccine

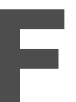

or the rational design of new efficacious and safe vaccines against tuberculosis (TB), it is essential to define and characterise the specificity of T-lymphocytes for mycobacterial antigens $(\mathrm{Ag})$, which can be related to protective or deleterious responses. Most studies of the human immune response to mycobacterial Ags have focused on blood cells [1-8]. TB, however, is predominantly a lung disease and local mucosal immune responses play an important role against invasion and subsequent spread of causative tubercle bacilli. Therefore, studies involving characterisation of the immune response to mycobacterial Ags from the actual site of infection are needed.

Limited information is available regarding the differences between mycobacterial Ags recognised by T-lymphocytes from peripheral blood and from the site of infection $[9,10]$. The capacity of different mycobacterial Ags to either activate or downregulate alveolar macrophages, the cells that primarily act as a habitat for tubercle bacilli, is also largely unknown. The present authors, however, have recently observed that the identical repertoire of mycobacterial Ags from the low molecular mass secretory proteome are recognised by peripheral blood mononuclear cells (PBMCs) of healthy TB contacts or treated TB (memory immune) subjects, and by pleural fluid mononuclear cells (PFMCs) of tuberculous pleurisy patients [11]. These findings led to the conclusion that considerable homogeneity exists between the specificity of PBMCs and PFMCs for mycobacterial Ags in human subjects representing a state of protective immunity. However, the lung and alveolar cavity represent the true sites of infection, as compared with pleura or peripheral blood, and to date no report is available regarding the fine specificity of bronchoalveolar

\section{AFFILIATIONS}

Depts of *Bochemistry and, \#Pulmonary Medicine, Postgraduate Institute of Medical Education \& Research, Chandigarh, India. "Mycobacteriology Laboratory Branch, Division of TB Elimination, Centers for Disease Control and Prevention, Atlanta, GA, USA.

\section{CORRESPONDENCE}

G.K. Khuller

Dept of Biochemistry

PGIMER

Chandigarh

160012

India

Fax: 991722744401

E-mail: gkkhuller@yahoo.co.in

Received:

September 232005

Accepted after revision:

September 202006

SUPPORT STATEMENT

This study was funded by a grant

from the Dept of Biotechnology and Indian Council of Medical Research, Government of India (New Delhi, India). S. B. Sable is the recipient of a Senior Research Fellowship of the Council of Scientific and Industrial Research (New Delhi, India).

STATEMENT OF INTEREST None declared. 
lavage (BAL) cells to mycobacterial Ags in TB subjects. Moreover, the comparative specificity of autologous blood and BAL cells to mycobacterial Ags has not been studied in detail. Therefore, in the present study, the local (BAL cell) immune response to mycobacterial Ags was compared with the systemic (peripheral blood) immune response in minimal TB patients.

\section{MATERIALS AND METHODS}

\section{Study subjects}

A total of 21 minimal TB patients and 10 non-TB patients with an indication for fiberoptic bronchoscopy were examined and diagnosed at the Dept of Pulmonary Medicine (PGIMER, Chandigarh, India) and used as study subjects. A chest radiography was obtained at the start of the study and the patients were graded as minimal TB patients according to the National Tuberculosis Association criteria [12]. In brief, patients classified as having minimal TB presented a low number of lesions on chest radiography, involving no more than one segment of lung, and usually had milder symptoms for a shorter period of time than patients with severe disease [4]. Active pulmonary TB was confirmed by positive sputum smear or sputum/BAL culture for acid-fast bacilli. Exclusion criteria for TB patients included disseminated or advanced TB, other pulmonary diseases or associated immunocompromised conditions, including HIV infection and the start of antituberculous drug therapy. The non-TB group included patients suffering from oesophagitis or haemoptysis of unknown aetiology. These subjects did not show any confirmed clinical, radiological or microbiological evidence of TB. Exclusion criteria for non-TB patients included pulmonary diseases, such as bronchial asthma or upper respiratory tract infections in the preceding 8 weeks, immunosuppressive diseases or medication. Although 10 non-TB patients participated in the study, data regarding lymphocyte proliferation and interferon (IFN)- $\gamma$ assays are only available for nine of them. Conversely, all 10 non-TB patients were evaluated in the nitric oxide (NO) assay. All the study subjects received bacilli Calmette-Guerin (BCG) as childhood vaccination and were from the north Indian states with high incidence of the disease. The Mantoux tests were not performed due to the poor specificity of the test in this population and the unwillingness of most donors to remain at the clinic for several days for the tests to be read. All the nonTB patients had no known contact with TB patients. However, $33.3 \%$ (three out of nine) and $66.6 \%$ (six out of nine) of non-TB patients responded to purified protein derivative (PPD) in an in vitro lymphocyte proliferation assay employing BAL cells and PBMCs, respectively, and their exposure to environmental mycobacteria is not known. However, widespread exposure to environmental mycobacterial species in India and in this region is well hypothesised for the failure of BCG vaccination and interference with the response to Mycobacterium tuberculosis [13]. Bronchoscopy [14] and venipuncture were carried out after informed written consent was obtained from all the study subjects and the ethics committee of the Postgraduate Institute of Medical Education and Research (Chandigarh, India) approved the study.

\section{Isolation of BAL cells and PBMCs}

Bronchoscopy was performed using an Olympus fibreoptic bronchoscope as previously described elsewhere [14], as part of the confirmatory procedure for TB. Sterile isotonic saline $(100 \mathrm{~mL})$ was instilled into the lungs after local anaesthesia of the upper airways with $2 \%$ lignocaine. The percentage of retrieved saline was mean \pm SEM $70 \pm 10 \%$ in both groups. The bronchoalveolar cells were obtained from BAL as described elsewhere [9, 15]. The cellular profiles of BAL cells in TB and non-TB patients are shown in table 1 . Venous blood was obtained from all the study subjects and PBMCs were isolated by centrifugation of heparinised blood layered over Ficoll-Hypaque. BAL cells and PBMCs were adjusted to $1 \times 10^{6}$ cells $\cdot \mathrm{mL}^{-1}$ after determining the viability of cells using the trypan-blue exclusion method.

\section{Antigens}

Total culture filtrate proteins of $M$. tuberculosis $\mathrm{H}_{37} \mathrm{Rv}$ (RvCFP) were obtained by stationary growth of tubercle bacilli in liquid modified Youman's medium [16] for 4 weeks. In a previous study [11], 104 purified mycobacterial polypeptides $(<40 \mathrm{kDa})$ were evaluated for the immune response induced in healthy TB contacts. Based on the type of immune response, immunodominant polypeptides were divided in two groups: group I, which consisted of 18 low molecular weight polypeptides prominently recognised by peripheral blood Tlymphocytes; and group II, which consisted of 10 low molecular weight polypeptides recognised by both T- and Blymphocytes of healthy TB contacts [11]. All the individual polypeptides used in the study were purified using combination of the anion exchange column chromatography and the high-resolution preparative sodium dodecyl sulphate polyacrylamide gel electrophoresis followed by electroelution [11]. Their characteristics are described in table 2.

\section{Lymphocyte proliferation assay}

BAL cells and PBMCs were added to flat-bottom 96-well tissue culture plates (Greiner Bio-one, Frickenhausen, Germany) at $1 \times 10^{5}$ cells $\cdot$ well $^{-1}$ in RPMI-1,640 medium (Sigma, St. Louis, MO, USA) supplemented with $50 \mu \mathrm{g} \cdot \mathrm{mL}^{-1}$ gentamicin sulphate, $200 \mathrm{mM}$ L-glutamine, $5 \times 10^{-5} \mathrm{M} \beta$-mercaptoethanol, $25 \mathrm{mM}$ hydroxyethyl piperazine ethane sulphonic acid (Fluka,

TABLE 1 Cellular profile of bronchoalveolar lavage (BAL) preparations in minimal tuberculosis (TB) and non-TB patients

\begin{tabular}{lccc} 
Group & Alveolar macrophages & Alveolar lymphocytes & Alveolar neutrophils \\
\hline TB patients \% & $58 \pm 3$ & $30 \pm 5$ & $12 \pm 5$ \\
Non-TB patients \% & $88 \pm 4$ & $10 \pm 2$ & $1 \pm 0$ \\
\hline
\end{tabular}

Data are presented as mean \pm SEM. The proportion of cells within BAL preparations was characterised morphologically according to Wright's stain. 
TABLE 2 Characterisation of group I and II purified polypeptides

\begin{tabular}{|c|c|c|}
\hline Serial No. & Apparent molecular weight kDa & Identity ${ }^{\#}$ \\
\hline \multicolumn{3}{|l|}{ Group I } \\
\hline 1 & 4.5 & Unknown \\
\hline 3 & 4.5 & TB10.4 Rv0288 \\
\hline 4 & 6.0 & ESAT-6 ${ }^{\pi},+$ Rv3875 \\
\hline 5 & 7.0 & ESAT-6 family member?" ${ }^{\bullet+}$ \\
\hline 8 & 9.5 & ESAT-6 family member?" \\
\hline 9 & 10.0 & CFP-10" Rv3874 \\
\hline 10 & 11.0 & Unknown \\
\hline 11 & 15.0 & MTSP17 Rv0164 \\
\hline 12 & 5.5 & Unknown \\
\hline 17 & 16.0 & MTSP14/CFP-17 $7^{\S}$ Rv1827 \\
\hline 18 & 18.0 & CFP-18 (mixed sample) $^{+}$Rv2185C, Rv1827 \\
\hline \multicolumn{3}{|l|}{ Group II } \\
\hline 19 & 22.5 & $\begin{array}{l}\text { CFP22. } 5^{5} \text { Mixed sample (Enoyl-CoA-hydratase, two component response regulator) } \\
\text { Rv0632C, Rv1626 }\end{array}$ \\
\hline 20 & $21-22.0$ & CFP-21 (cutinase precursor) ${ }^{\circ}$ Rv1984C \\
\hline 21 & 19.0 & Unknown \\
\hline 22 & 24.0 & MPT-64", +, § Rv1980C \\
\hline 23 & 30.5 & Ag85A (MPT-44, mycolyl transferase) ${ }^{\uparrow,+,}$, Rv3804C \\
\hline 24 & 29.5 & Ag85B (MPT-59, mycolyl transferase) ${ }^{\tau,+,}{ }^{f}$ Rv1886C \\
\hline 25 & 31 & CFP-31 $1^{\S}$ (hypothetical protein) ${ }^{+}, f$ Rv0831C \\
\hline
\end{tabular}

ESAT: early secreted antigenic target; TB: tuberculosis; CFP: culture filtrate protein; MTSP: Mycobacterium tuberculosis secretory protein; Ag: antigen; MPT Mycobacterium tuberculosis protein. ${ }^{*}$ : identified using [17]; ": identity determined by monoclonal/polyclonal antibody reactivity; ${ }^{+}$: identity determined by liquid chromatography-mass spectrometry (MS)-MS analysis; ${ }^{5}$ : identified previously as novel T- cell antigens $[11,18] ;{ }^{f}$ : identity determined by $\mathrm{N}$-terminal sequencing.

Buchs, Switzerland), $1 \mathrm{mM}$ sodium pyruvate and $10 \%$ heatinactivated autologous serum. Selected purified polypeptides $\left(2 \mu \mathrm{g} \cdot \mathrm{mL}^{-1}\right), \mathrm{RvCFP}, \mathrm{PPD}\left(2 \mu \mathrm{g} \cdot \mathrm{mL}^{-1}\right)$ and the mitogen phytohaemagglutinin $\left(1 \mu \mathrm{g} \cdot \mathrm{mL}^{-1}\right)$ were used for in vitro stimulation. Cultures were incubated for 5 days at $37^{\circ} \mathrm{C}$ in an atmosphere containing $5 \% \mathrm{CO}_{2}$ and $100 \%$ humidity. At the end of the incubation period, $0.25 \mu \mathrm{Ci}{ }^{3} \mathrm{H}$-thymidine was added followed by incubation for $18-22 \mathrm{~h}$. The cells were harvested on to glass fibre filters using the Nunc cell harvester (Intermed, Roskilde, Denmark) and the incorporated radioactivity was measured in an LKB Rackbeta liquid scintillation counter (model 1214; LKB-Wallac, Palo Alto, CA, USA). The proliferation responses were expressed as stimulation indices (SI). SI were calculated by dividing mean counts per minute in Ag-stimulated wells by mean counts per minute in unstimulated wells. A SI value of 3.0 was used as a positive cut-off point to determine the percentage recognition of different mycobacterial Ags by PBMCs and BAL cells.

\section{IFN- $\gamma$ and interleukin-12 (p40) assay}

The levels of IFN- $\gamma$ released by PBMCs and BAL cells in response to the individual $\mathrm{Ag}$ were estimated in culture supernatants collected on day 5 using a human IFN- $\gamma$ reagent set (Opt EIA ${ }^{\mathrm{TM}}$ set; BD Pharmingen, San Diego, CA, USA). The assay was performed according to the manufacturer's instructions. Quantitative interleukin (IL)-12 (p40) measurements were performed similarly with $24 \mathrm{~h}$ supernatants from PBMC and BAL cell cultures using human IL-12 (p40) reagents (Duoset R\&D system; Becton, Dickinson \& Co. Biosciences Pharmingen, San Diego, CA, USA). The lower limit of detection for IFN- $\gamma$ was $2.35 \mathrm{pg} \cdot \mathrm{mL}^{-1}$ and for IL-12 (p40) it was $16.0 \mathrm{pg} \cdot \mathrm{mL}^{-1}$. 


\section{NO assay}

BAL cells $\left(1 \times 10^{5}\right.$ cells $\cdot$ well $\left.^{-1}\right)$ were cultured for different time intervals ranging from day 0 to day 10 in phenol red-free Dulbecco's modified Eagle medium (Sigma) supplemented with $50 \mu \mathrm{g} \cdot \mathrm{mL}^{-1}$ gentamicin sulphate, $200 \mathrm{mM}$ L-glutamine, $2.5 \mathrm{mM}$ L-arginine and $10 \%$ heat-inactivated autologous serum. M. tuberculosis RvCFP, PPD and selected purified polypeptides were used at a concentration of $2 \mu \mathrm{g} \cdot \mathrm{mL}^{-1}$, while Escherichia coli lipopolysaccharide (LPS) was used at a concentration of $10 \mu \mathrm{g} \cdot \mathrm{mL}^{-1}$ for the in vitro stimulation. NO, measured in terms of the amount of nitrite released in culture supernatants, was estimated using Griess reagent as described by GREEN et al. [19].

\section{Statistical analysis}

All results are presented as mean values of triplicate wells. The data obtained were analysed using the Mann-Whitney twotailed test and ANOVA. A p-value $<0.05$ was considered statistically significant.

\section{RESULTS}

\section{Lymphocyte responses of BAL cells and PBMCs to mycobacterial Ags}

Lymphocyte proliferation in response to group I (fig. 1) and group II (fig. 2) Ags was assessed in BAL cells and PBMCs from minimal TB and non-TB patients.

When the proliferative response of BAL cells of minimal TB patients to in vitro stimulation with group I Ags was evaluated, the polypeptides 2, 14, 3, 18 and 15 induced prominent lymphocyte proliferation (median SI $\geqslant 3.0$; fig. 1a). The maximum SI was observed for polypeptide 2, a putative $6 \mathrm{kDa}$ early secreted antigenic target (ESAT)- 6 family member (median SI 4.47, 58.82\% recognition; table 2). Although increased response was observed for all the polypeptides in TB patients, no statistically significant difference was observed when BAL cell responses of group I polypeptides in TB patients (fig. 1a) were compared with BAL cell proliferative responses of non-TB patients (fig. 1c). All group II polypeptides, except polypeptides 26 and 27 , induced prominent BAL

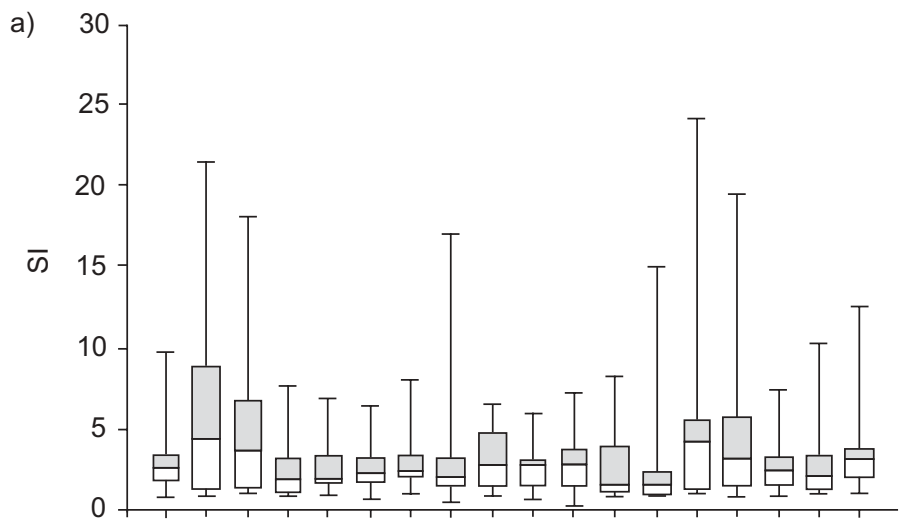

b)

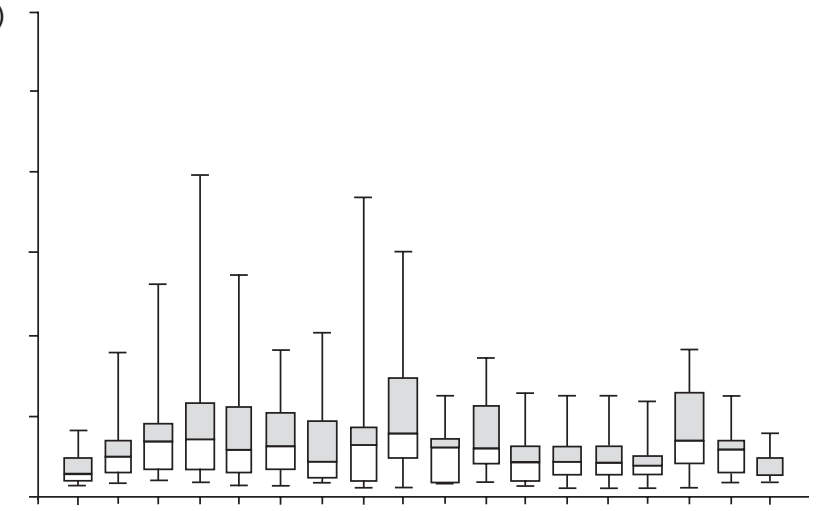

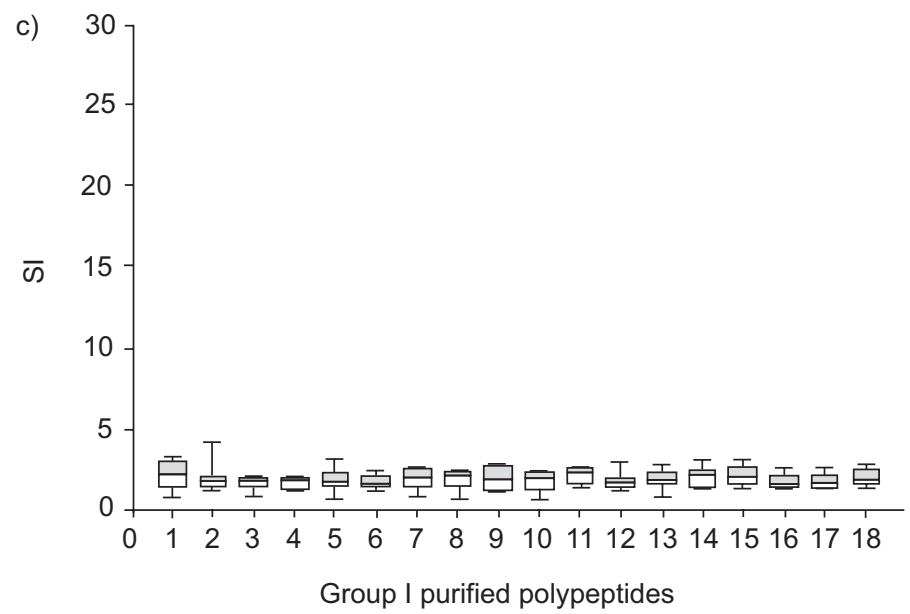

d)

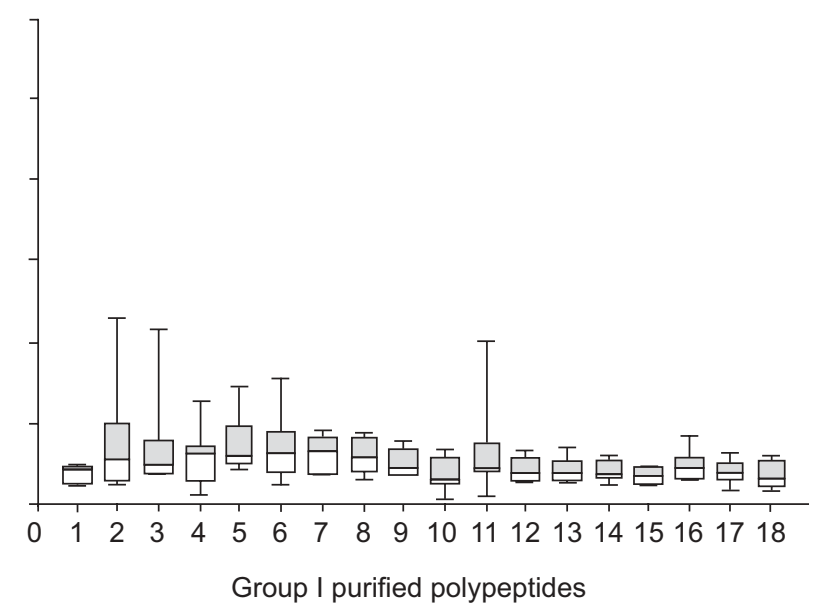

FIGURE 1. Proliferative responses of bronchoalveolar lavage (BAL) cells (a and c) and peripheral blood mononuclear cells (PBMCs; b and d) of minimal tuberculosis (TB; $a$ and b) and non-TB ( $c$ and d) patients to in vitro stimulation with group I low molecular mass purified polypeptides. Standard box plots with median (25th and 75th percentiles) and whiskers (at minimum and maximum values) are shown. The median counts per minute of BAL cell and PBMC cultures without antigen in TB patients was 780 and 650, while that in non-TB patients was 632 and 710, respectively. The median stimulation indices (SI) in response to phytohaemagglutinin of BAL cells and PBMCs of TB patients was 20.81 and 13.04, while that in non-TB patients was 14.22 and 16.0, respectively. 
a)

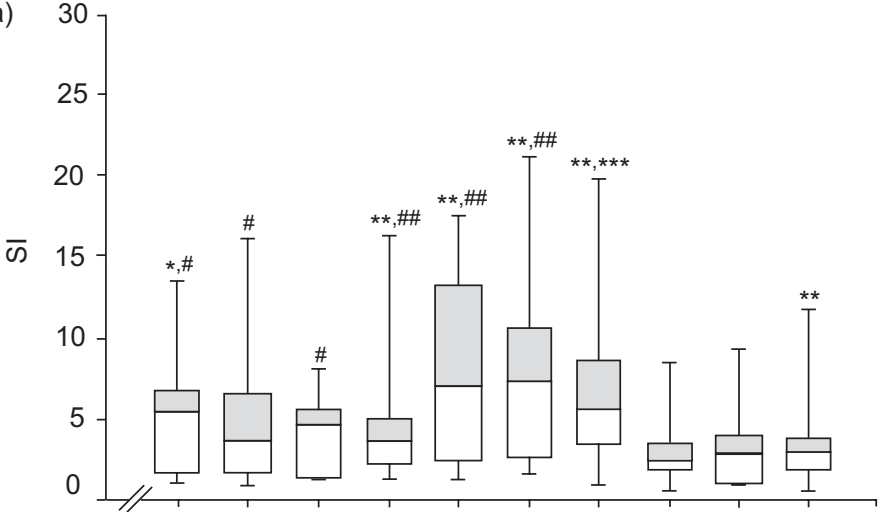

c)

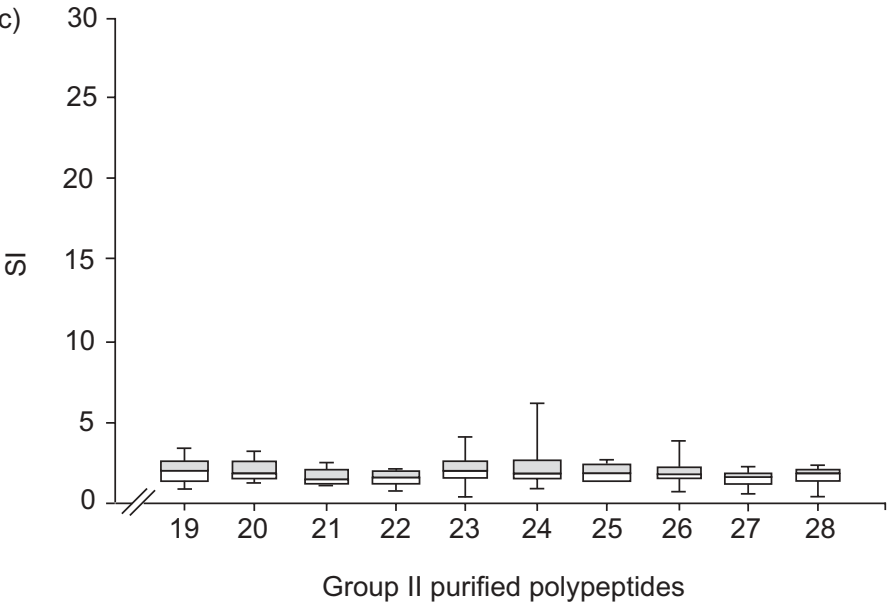

b)

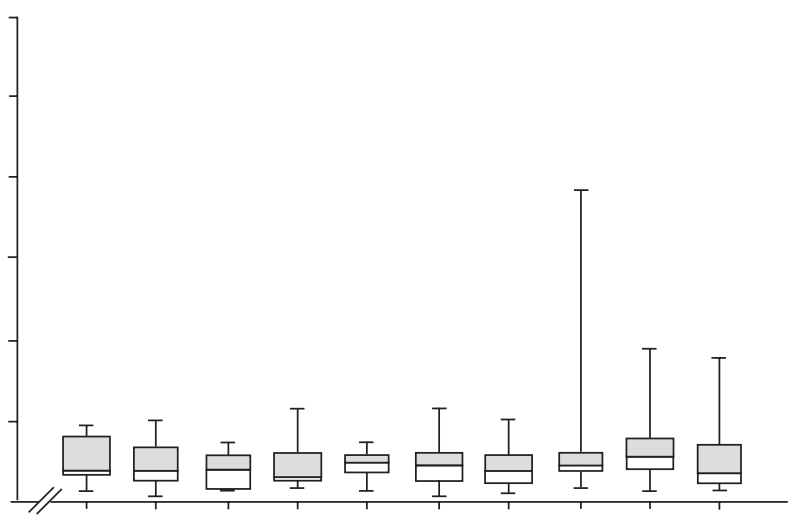

d)

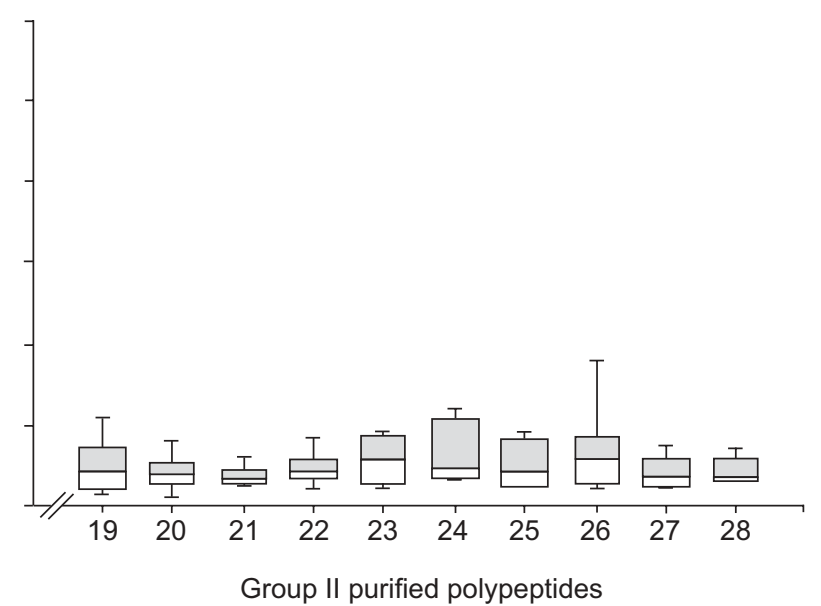

FIGURE 2. Proliferative responses of bronchoalveolar lavage (BAL) cells ( $a$ and $c$ ) and peripheral blood mononuclear cells (PBMCs; $b$ and d) of minimal tuberculosis (TB; $a$ and b) and non-TB ( $\mathrm{c}$ and d) patients to in vitro stimulation with group II low molecular mass purified polypeptides. Standard box plots with median (25th and 75th percentiles) and whiskers (at minimum and maximum values) are shown. The median stimulation indices (SI) in response to total culture filtrate proteins of Mycobacterium tuberculosis $\mathrm{H}_{37} \mathrm{Rv}$ of BAL cells and PBMCs of TB patients was 12.44 and 6.13, while that in non-TB patients was 3.81 and 6.0 , respectively. The median SI in response to purified protein derivative of BAL cells and PBMCs of TB patients was 8.22 and 4.40 , while that in non-TB patients was 2.44 and 5.8 , respectively. ${ }^{*}: p<0.05$; ${ }^{* *}: p<0.01$ versus BAL, non-TB; and ***: $p<0.001 ;{ }^{*}: p<0.05 ;{ }^{\# \#: ~} p<0.01$ versus PBMC, TB.

cell proliferation (median SI $\geqslant 3.0$; fig. $2 \mathrm{a}$ ) and the maximum SI was observed in response to polypeptide 24 (Ag85B, median SI $7.21,70.58 \%$ recognition; table 2 ). Significantly high proliferative response in BAL cells of TB patients (fig. 2a), as compared with non-TB patients (fig. 2c), was observed for polypeptides 22 (Mycobacterium tuberculosis protein (MPT)-64), 24 (Ag85B), 23 (Ag85A), 25 (culture filtrate protein (CFP)-31), 28 (CFP-11; $\mathrm{p}<0.01)$ and polypeptide 19 (CFP-22.5; $\mathrm{p}<0.05)$.

When the proliferative response of PBMCs of TB patients to stimulation with group I Ags was evaluated, the top five polypeptides, which induced prominent lymphocyte proliferation, were polypeptides 9, 4, 3, 16 and 6 (median SI >3.0; fig. 1b). A maximum SI value was observed for polypeptide 9 (CFP-10; median SI 3.84, 70.58\% recognition; table 2). Conversely, when the PBMC response to group II polypeptides was evaluated, none induced prominent lymphocyte proliferation (median SI $\geqslant 3.0$; fig. $2 b$ ) and the maximum SI was observed in response to polypeptide 27 (CFP-12.5; median SI $2.86,47.05 \%$ recognition; table 2 ).
When the proliferative responses of PBMCs and BAL cells of TB patients to group I polypeptides were compared, no significant difference was observed. However, the BAL cell responses of TB patients were significantly high compared with PBMC responses for polypeptides 25 (CFP-31; $\mathrm{p}<0.001)$, 22 (MPT-64), 24 (Ag85B), 23 (Ag85A; $\mathrm{p}<0.01), 19$ (CFP-22.5), 20 $(\mathrm{CFP}-21)$ and $21(\mathrm{p}<0.05$; fig. $2 \mathrm{a}$ and $\mathrm{b})$ from group II polypeptides. Comparison of the proliferative responses of BAL cells and PBMCs of non-TB patients demonstrated no significant difference for the majority of mycobacterial Ags evaluated (fig. 1c, $1 \mathrm{~d}, 2 \mathrm{c}$ and $2 \mathrm{~d}$ ).

IFN- $\gamma$ levels in response to group I (fig. 3) and group II (fig. 4) Ags were estimated in BAL cells and PBMCs culture supernatants from minimal TB and non-TB patients. BAL cells of TB patients were found to release significantly higher levels $(\mathrm{p}<0.01$ to $\mathrm{p}<0.001)$ of IFN- $\gamma$ in in vitro culture supernatants than those of non-TB patients, in response to all mycobacterial Ags tested (fig. 3a, 3c, 4a and 4c). Among the group I polypeptides evaluated, polypeptides 9 (CFP-10), 4 (ESAT-6), 

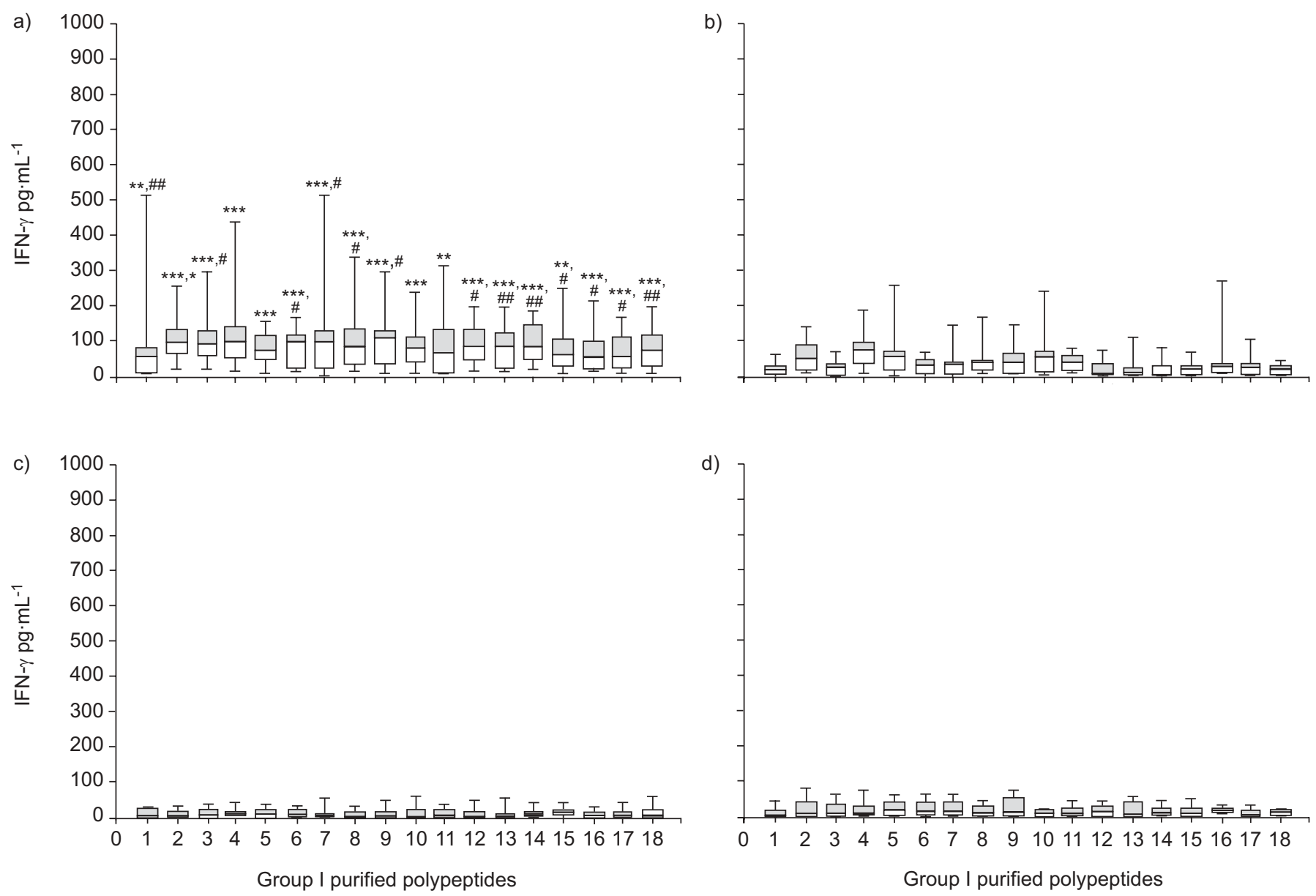

FIGURE 3. Interferon (IFN)- $\gamma$ responses of bronchoalveolar lavage (BAL) cells (a and c) and peripheral blood mononuclear cells (PBMCs; $b$ and d) of minimal tuberculosis (TB; $a$ and b) and non-TB (c and d) patients to group I low molecular mass purified polypeptides. Standard box plots with median (25th and 75th percentiles) and whiskers (at minimum and maximum values) are shown. The median IFN- $\gamma$ released in BAL cell and PBMCs cultures without antigen was $9.0 \mathrm{pg} \cdot \mathrm{mL}^{-1}$ and below the detection limit (BDL) in TB patients, while that in non-TB patients was $4.0 \mathrm{pg} \cdot \mathrm{mL}^{-1}$ and BDL, respectively. The median IFN- $\gamma$ released in response to phytohaemagglutinin in BAL cells and PBMC cultures of TB patients was 440.0 and $272.0 \mathrm{pg} \cdot \mathrm{mL}^{-1}$, while that in non-TB patients was 264.0 and $360.0 \mathrm{pg} \cdot \mathrm{mL}^{-1}$, respectively. ${ }^{\star *}: \mathrm{p}<0.01 ;{ }^{* \star *}: \mathrm{p}<0.001$ versus BAL, non-TB; and *: $p<0.05$; ${ }^{*}: \mathrm{p}<0.01$; ${ }^{\# \#: ~} \mathrm{p}<0.001$ versus PBMC, TB.

6 (CFP-8), 7 and 2 (both putative ESAT-6 family members) were found to induce prominent IFN $-\gamma$ release (median IFN- $\gamma$ $\geqslant 100.0 \mathrm{pg} \cdot \mathrm{mL}^{-1}$; fig. 3a) in BAL cell culture supernatants of TB patients, with a maximum IFN- $\gamma$ release observed in response to polypeptide 9 (CFP-10; median IFN- $\left.\gamma 110.0 \mathrm{pg} \cdot \mathrm{mL}^{-1}\right)$. When group II polypeptides were evaluated, polypeptides 24 (Ag85B), 23 (Ag85A), 22 (MPT-64), 19 (CFP-22.5), 20 (CFP-21), 27 (CFP-12.5) and 28 (CFP-11) were found to induce prominent IFN- $\gamma$ release (median IFN- $\gamma \geqslant 100.0 \mathrm{pg} \cdot \mathrm{mL}^{-1}$ ) in BAL cell culture supernatants, with a maximum response observed to polypeptide 24 (Ag85B; median IFN- $\gamma 240.0 \mathrm{pg} \cdot \mathrm{mL}^{-1}$; fig. $\left.4 \mathrm{a}\right)$.

In the case of PBMCs of TB patients, a maximum IFN- $\gamma$ release in culture supernatant was observed for polypeptide 4 (ESAT6; median IFN- $\gamma 78.0 \mathrm{pg} \cdot \mathrm{mL}^{-1}$ ) from group I (fig. 3b) and for polypeptide 24 (Ag85B; median IFN- $\gamma 45.0 \mathrm{pg} \cdot \mathrm{mL}^{-1}$ ) from group II (fig. $4 \mathrm{~b}$ ). The IFN- $\gamma$ responses of BAL cells of TB patients were significantly higher $(p<0.05$ to $p<0.001)$ when compared with those induced by PBMCs in response to all Ags evaluated. The exceptions were polypeptides 4 (ESAT-6), 5 (putative ESAT-6 family member), 10 (uncharacterised) and 11
(Mycobacterium tuberculosis secretory protein (MTSP)-17; fig. 3a, 3b, 4a and 4b). No significant difference was observed for group I and group II Ags when IFN- $\gamma$ responses of BAL cells and PBMCs of non-TB patients were compared (fig. 3c, $3 \mathrm{~d}, 4 \mathrm{c}$ and $4 \mathrm{~d})$.

Though not statistically significant, the proliferative and IFN- $\gamma$ responses of BAL cells of minimal TB patients to stimulation with group II Ags were high when compared with group I Ags. In group I, five out of 18 polypeptides demonstrated median SI in the range 3.0-5.0 and median IFN- $\gamma$ in the range $100.0-150.0 \mathrm{pg} \cdot \mathrm{mL}^{-1}$. However, in group II, eight out of 10 polypeptides induced median SI in the range 3.0-7.5 and six out of 10 polypeptides induced median IFN- $\gamma$ in the range of $100.0-250.0 \mathrm{pg} \cdot \mathrm{mL}^{-1}$. Conversely, although not statistically significant, there was a trend in PBMCs of TB patients toward increased recognition of the majority of group-I Ags when compared with group-II Ags. In group I, seven out of 18 polypeptides demonstrated median SI $>3.0$ and four out of 18 polypeptides demonstrated median IFN- $\gamma$ responses in the range $50.0-100 \mathrm{pg} \cdot \mathrm{mL}^{-1}$. However, in group II, all 10 polypeptides 

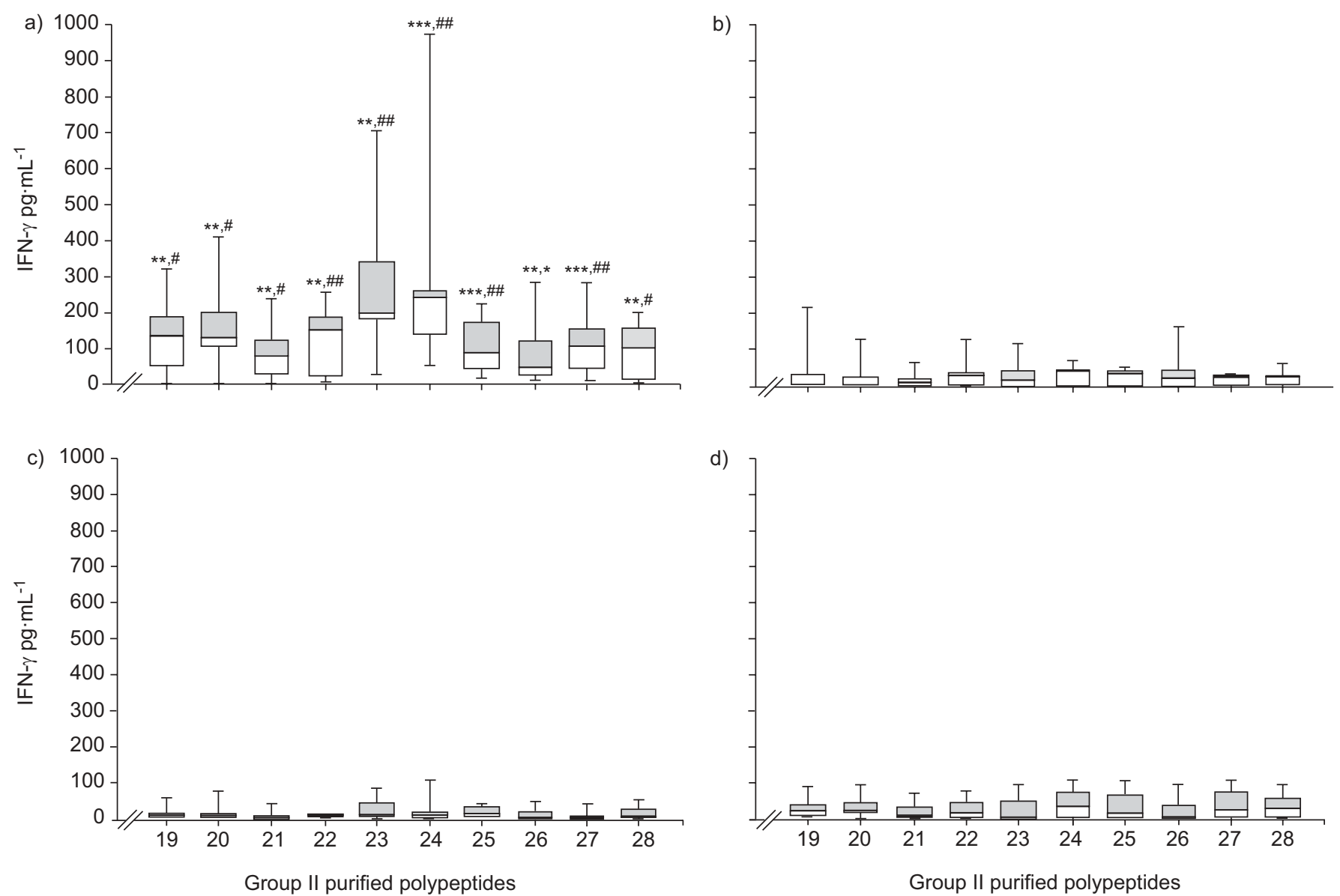

d)

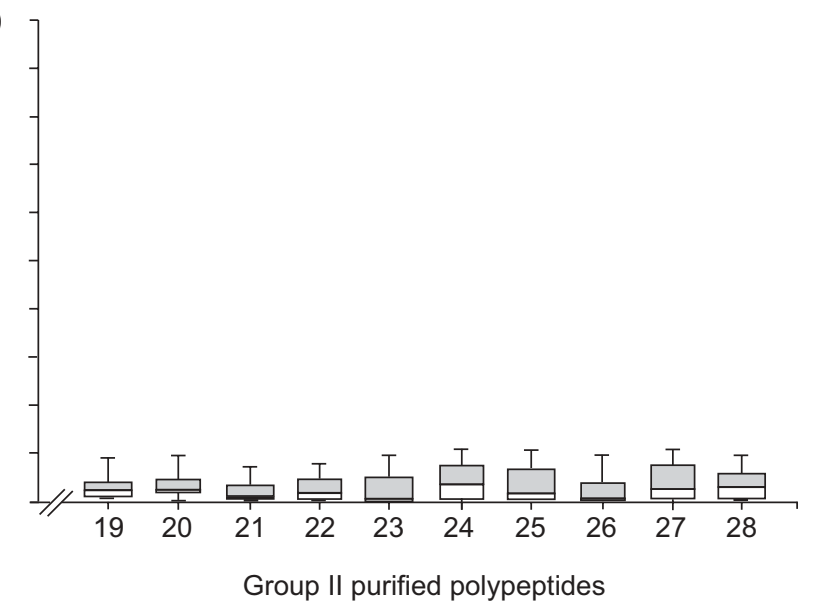

FIGURE 4. Interferon (IFN)- $\gamma$ responses of bronchoalveolar lavage (BAL) cells (a and c) and peripheral blood mononuclear cells (PBMCs; b and d) of minimal tuberculosis (TB; $a$ and b) and non-TB (c and d) patients to group II low molecular mass purified polypeptides. Standard box plots with median (25th and 75th percentiles)

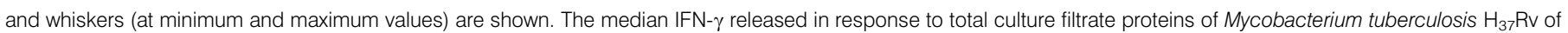
BAL cells and PBMC cultures of TB patients was 190.0 and $69.0 \mathrm{pg} \cdot \mathrm{mL}^{-1}$, while that in non-TB patients was 44.0 and $64.0 \mathrm{pg} \cdot \mathrm{mL}^{-1}$, respectively. The median IFN- $\gamma$ released in response to purified protein derivative in BAL cell and PBMC culture supernatants of TB patients was 190.0 and $58.0 \mathrm{pg} \cdot \mathrm{mL}^{-1}$, while that in non-TB patients was 28.0 and $54.0 \mathrm{pg} \cdot \mathrm{mL}^{-1}$, respectively. ${ }^{* *}: \mathrm{p}<0.01 ;{ }^{* *}: \mathrm{p}<0.001$ versus BAL, non-TB; and ${ }^{*}: \mathrm{p}<0.05 ;{ }^{*}: \mathrm{p}<0.01 ;{ }^{* \#}: \mathrm{p}<0.001$ versus PBMC, TB.

induced median SI $<3.0$ with median IFN- $\gamma$ responses $<50.0 \mathrm{pg} \cdot \mathrm{mL}^{-1}$.

\section{Macrophage responses of BAL cells and PBMCs to mycobacterial Ags}

The activating ability of groups I and II CFPs over alveolar macrophages and blood monocytes was evaluated by estimating the levels of NO and IL-12 (p40) in the culture supernatants of BAL cells and PBMCs, respectively. To determine the kinetics of NO release in culture supernatants of BAL cells in response to in vitro stimulus, BAL cells from active minimal TB patients were cultured with different stimuli, i.e. M. tuberculosis $\mathrm{H}_{37} \mathrm{Ra}\left(5: 1 \mathrm{M}\right.$. tuberculosis:BAL cells), RvCFP $\left(10 \mu \mathrm{g} \cdot \mathrm{mL}^{-1}\right)$ and E. coli LPS $\left(10 \mu \mathrm{g} \cdot \mathrm{mL}^{-1}\right)$ for a varying period of time. NO levels, measured in terms of nitrite release, were negligible at initial periods of 24 and $48 \mathrm{~h}$. Levels of nitrite release in response to RvCFP were found to be low when compared with whole bacilli (data not shown). Since significant levels of nitrite were observed by day 7; supernatants collected at this time-point were used for the nitrite estimation in subsequent studies. Conversely, studies carried out using blood monocyte-derived macrophages produced negligible NO (nitrite) in culture supernatants $(<4 \mu \mathrm{M})$ when $M$. tuberculosis $\mathrm{H}_{37} \mathrm{Rv}$ CFPs or purified polypeptides were used as in vitro stimulus (data not shown). Figure 5 depicts NO (nitrite) levels released by BAL cells of TB and non-TB patients in response to group I and group II polypeptides.

When NO responses of BAL cells of TB patients to stimulation with group I Ags were evaluated, the polypeptides 14, 5, 8, 4, 9 and 17 induced prominent NO release (median NO $>5.0 \mu \mathrm{M}$; fig. 5a). The maximum NO production was observed in response to polypeptide 14 , an as yet uncharacterised $\mathrm{Ag}$ (median NO 6.29). No statistically significant difference was observed for the majority of group I polypeptides to induce nitrite in BAL cell cultures of $\mathrm{TB}$ and non-TB patients. However, for polypeptides 2, 10, 11 (CFP-11) and 13, high nitrite response was observed in BAL cell cultures of non-TB subjects $(p<0.01)$ as compared with that of TB patients, suggesting an increased alveolar macrophage response for the aforementioned polypeptides in non-TB patients (fig. 5a and $b$ ). When NO responses of group II Ags were evaluated in $\mathrm{TB}$ patients, a prominent nitrite release was observed for all the 


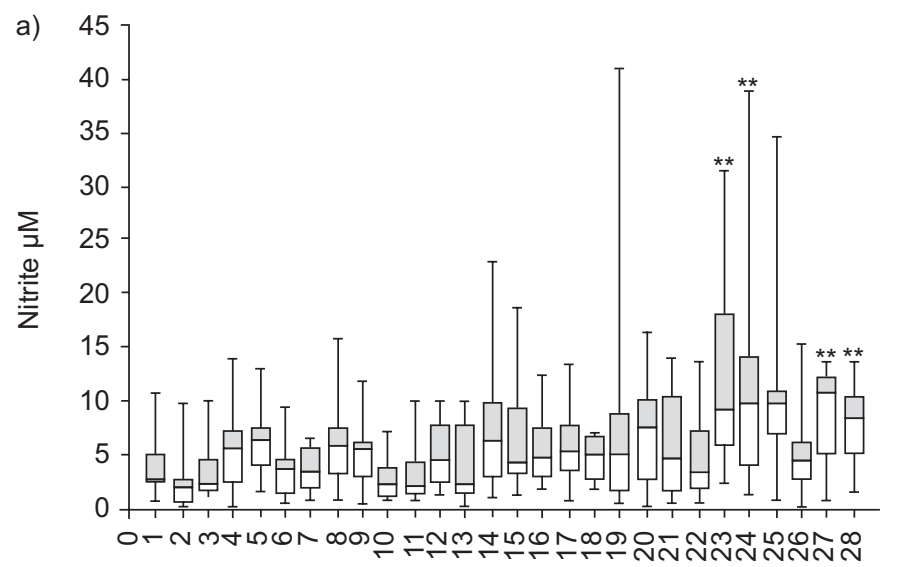

Group I and II purified polypeptides

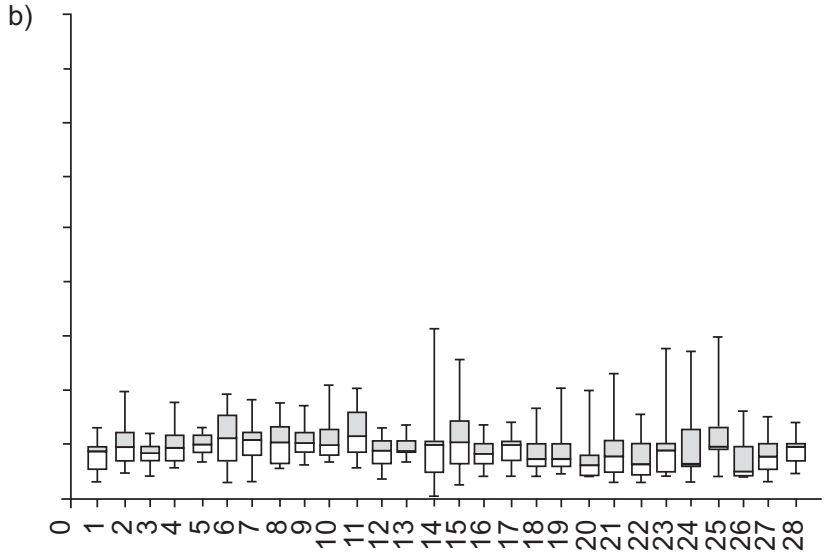

Group I and II purified polypeptides

FIGURE 5. Nitric oxide (NO) responses of bronchoalveolar lavage (BAL) cells of minimal tuberculosis (TB; a) and non-TB (b) patients induced by group I and group II low molecular mass purified polypeptides. Standard box plots with median (25th and 75th percentiles) and whiskers (at minimum and maximum values) are shown. The median

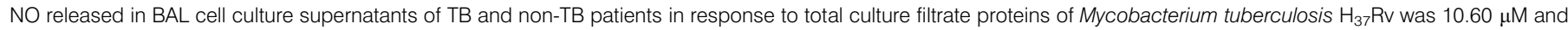
$6.28 \mu \mathrm{M}$, while that released in response to purified protein derivative was $11.71 \mu \mathrm{M}$ and $5.95 \mu \mathrm{M}$, respectively. The median NO levels released in BAL cell culture supernatants of TB and non-TB patients in response to medium alone were $2.90 \mu \mathrm{M}$ and $2.79 \mu \mathrm{M}$, respectively. ${ }^{*}$ : $\mathrm{p}<0.01$ versus BAL, non-TB.

polypeptides except 21, 22 and 26 (median $\mathrm{NO}>5.0 \mu \mathrm{M}$; fig. 5a). The maximum NO release was induced by polypeptide 27, a mixed sample (median NO 10.80), followed by polypeptide 24 (Ag85B; median NO 9.79). However, levels of nitrite release were found to be significantly higher in BAL cell cultures of TB patients for polypeptides 24 (Ag85B), 23 (Ag85A), 27 (CFP-12.5) and 28 (CFP-11; p<0.01) when compared with those induced in BAL cell cultures of non-TB patients (fig. 5).

Group II polypeptides were found to release high levels of nitrite in BAL cell cultures of minimal TB patients when compared with group-I polypeptides. Among group II polypeptides, seven out of 10 demonstrated high nitrite responses $>5.0 \mu \mathrm{M}$ (median range $3.49-10.80 \mu \mathrm{M}$ ), while only six out of 18 group I polypeptides induced nitrite levels $>5.0 \mu \mathrm{M}$ $(2.00-6.29 \mu \mathrm{M})$

Simultaneously, BAL cells and PBMCs of minimal TB and nonTB patients were stimulated with mycobacterial Ags for $24 \mathrm{~h}$. IL-12 levels in culture supernatants were measured by sandwich ELISA. IL-12 (p40) levels above the detection limit were observed only for RvCFP, PPD, Ag85B and Ag85A in BAL cell cultures of minimal TB patients. None of the mycobacterial Ags tested induced significant IL-12 (p40) release in BAL cells or PBMCs culture supernatants of nonTB patients (data not shown). Thus, the present study demonstrates the increased recognition of Ag85 complex (A and B) polypeptides by BAL cells of minimal TB patients and this is made evident by high lymphoproliferative, IFN- $\gamma$, NO and IL-12 responses.

\section{DISCUSSION}

The differences in mycobacterial Ag specificity of lung cells derived from bronchoalveolar spaces and mononuclear cells isolated from peripheral venous blood of human subjects have not been evaluated. Therefore, in the present study, 18 predominant T-cell (group I) and $10 \mathrm{~T}$ - and B-cell (group II) response-inducing polypeptides recognised by PBMCs of healthy TB contacts and PFMCs of tuberculous pleurisy patients [11] were investigated. Due to the ethical constraints of bronchoscopy of healthy TB contacts, a human model of protective immunity, and due to non responsiveness of BAL cells to M. tuberculosis Ags in healthy human subjects [9, 10], the present authors evaluated mycobacterial Ags for their recognition by BAL cells of minimal TB patients demonstrating least pathology. The advanced TB patients were not investigated due to their extensive lung pathology and peripheral anergy, indicative of diminished operative beneficial responses. ${ }^{3} \mathrm{H}$-thymidine uptake assay (DNA synthesis) using BAL cells from radiologically affected lung of minimal TB patients demonstrated increased responsiveness to the majority of mycobacterial Ags evaluated. It was associated with increased IFN- $\gamma$ secretion in in vitro cultures. In contrast, PBMCs from the same TB patients were generally hyporesponsive to these stimuli in terms of IFN- $\gamma$ production, with the exception of ESAT-6, MTSP-17 and polypeptides 5 (putative ESAT-6 family member) and 10 (uncharacterised), where no statistically significant difference between the BAL and PBMC responses was observed. Increased proliferative and IFN- $\gamma$ responses of BAL cells of TB patients over those of non-TB patients to in vitro stimulation of mycobacterial Ags indicate mycobacterial Ag-specific hyperresponsiveness of BAL cells of TB patients. The disparity between specific cell count in BAL cell preparations of TB and non-TB patients (table 1) might be suspected to account for these differences. Though data is not corrected for these differences in the present study, the cell number adjusted enzyme-linked immunospot assay in a study by SCHWANDER et al. [9] indeed suggested that there is an absolute increase in $M$. tuberculosis Ag-specific IFN- $\gamma$ secreting cells in BAL of TB patients compared with control subjects. Since IFN- $\gamma$ is generally produced by lymphocytes, and not other cells, the results 
support the likelihood that the mycobacterial Ags induced increased stimulation indices and this could not be accounted for only by an increase in the numbers of lymphocytes. The present observations of such compartmentalisation of antimycobacterial immune responses at the site of infection during active pulmonary TB are in accordance with those reported by others $[9,10]$. This may be due to localisation of circulating specific cells or local proliferation and expansion of few specific precursors or a combination of the two mechanisms.

In the present study, although responses of group I and II polypeptides were not statistically different, the majority of group II polypeptides were found to be prominently recognised by BAL cells of minimal TB patients in terms of both lymphocyte proliferation and IFN- $\gamma$ induction. A predominant responsiveness was observed to Ag85B, Ag85A, CFP-31, CFP21, CFP-22.5, MPT-64 and the as yet uncharacterised 19-kDa protein. The reasons for such an increased recognition of group II Ags by BAL cells are not clear, but can be attributed to the strong inherent immunogenicity of these polypeptides $[18,20]$. The polypeptide recognition pattern of BAL cells of minimal TB patients observed in the present study was found to be different from that of PBMCs of healthy TB contacts and PFMCs of tuberculous pleurisy patients, where an increased recognition of group I polypeptides was observed [11]. Furthermore, a more pronounced diversity was observed in the Ag recognition by autologous PBMCs and BAL cells of minimal TB patients. Nonetheless, the disparity between spleen and lung immune responses in mice [21, 22], as well as between PBMCs and BAL cell responses in humans using few characterised Ags like ESAT-6 and Ag85 complex proteins, have been reported previously $[9,10]$. This is consistent with the observation made in the present study.

Apart from alveolar lymphocytes, alveolar macrophages have been shown to play an important role in the defense against $M$. tuberculosis $[15,23]$. The capacity of alveolar macrophages obtained from affected lung of minimal TB patients to produce NO and IL-12 in response to in vitro stimulation with $M$. tuberculosis and its secretory Ags was investigated and compared with that obtained in blood monocytes. Some studies failed to show production of NO by human macrophages [24, 25], while numerous other studies successfully demonstrated the capacity of human macrophages to produce NO $[23,26]$. In the present study, alveolar macrophages, and not blood monocytes, were capable of producing NO after in vitro stimulation with $M$. tuberculosis and its secretory Ags. It is thus demonstrated that alveolar macrophages activated with various stimuli failed to produce $\mathrm{NO}$ at early time-points of 24-48 $\mathrm{h}$ and NO production was seen only after several days of culture (4-7 days). It can be speculated that IFN- $\gamma$ produced by the alveolar lymphocytes after 72-96 h of stimulation might be responsible for their activation and subsequent release of detectable NO levels. It has been well documented that Agspecific lymphocytes enhance NO production by macrophages through increased IFN- $\gamma$ release $[27,28]$. This might be responsible for the differences in the NO levels observed in response to the different Ags screened. BAL cell responses to Ag85A and Ag85B proteins observed in the present study can be cited to explain the link between alveolar macrophage and lymphocyte responses. BAL cell responses to these Ags were characterised by high lymphocyte proliferation and prominent
IL-12 (p40), IFN- $\gamma$ and NO responses in minimal TB patients. In addition, detectable in vitro IL-12 (p40) responses to Ag85 complex proteins only were also observed in BAL cells of minimal TB patients. In fact, Ag85A and B proteins had already been demonstrated as suitable candidates for the development of future antituberculous vaccine [21, 29-31]. Predominant recognition of these proteins by BAL cells, characterised by prominent release of IL-12, IFN- $\gamma$ and NO in vitro, suggests the potential of these Ags as constituents of a future mucosal antiTB vaccine. The recent observations of improved protection by these vaccine candidates following intranasal vaccination of mice against $M$. tuberculosis challenge $[32,33]$ and their entry into clinical trials $[30,34]$ bodes well for their use as mucosal vaccines for humans and needs further evaluation.

Thus, the overall data presented in the current study suggests that lymphocyte and macrophage responses at the actual site of disease differ to responses by the same cells in the periphery. Therefore, it seems imperative to evaluate vaccine candidates both at the level of the lung and blood cells for their recognition.

\section{ACKNOWLEDGEMENTS}

The current authors gratefully acknowledge the help of J.T. Belisle and K. Dobos (Mycobacterial Research Laboratories, Dept of Microbiology, Immunology and Pathology, C.S.U. Fort Collins, CO, USA) in LC-MS-MS analysis of mycobacterial antigens, as well as in providing MAbs from World Health Organization collection, and I. Rosenkrands (Statens Serum Institute, Copenhagen, Denmark) in providing monoclonal antibodies against region of difference (RD)1 and RD2 proteins. The authors would like to sincerely thank V. Singh for his help with the graphics and gratefully acknowledge the participation of all the volunteers in this study.

\section{REFERENCES}

1 Havlir DV, Wallis RS, Boom WH, Daniel TM, Chervenak K, Ellner JJ. Human immune response to Mycobacterium tuberculosis antigens. Infect Immun 1991; 59: 665-670.

2 Schoel B, Gulle H, Kaufmann SH. Heterogeneity of the repertoire of $\mathrm{T}$ cells of tuberculosis patients and healthy contacts to Mycobacterium tuberculosis antigens separated by high-resolution technique. Infect Immun 1992; 60: 1717-1720.

3 Boesen H, Jensen BN, Wilcke T, Andersen P. Human T-cell responses to secreted antigen fractions of Mycobacterium tuberculosis. Infect Immun 1995; 63: 1491-1497.

4 Demissie A, Ravn P, Olobo J, et al. T-cell recognition of Mycobacterium tuberculosis culture filtrate fractions in tuberculosis patients and their household contacts. Infect Immun 1999; 67: 5967-5971.

5 Skjot RL, Oettinger T, Rosenkrands I, et al. Comparative evaluation of low-molecular-mass proteins from $\mathrm{Myco-}$ bacterium tuberculosis identifies members of the ESAT-6 family as immunodominant T-cell antigens. Infect Immun 2000; 68: 214-220.

6 Alderson MR, Bement T, Day CH, et al. Expression cloning of an immunodominant family of Mycobacterium tuberculosis antigens using human CD4(+) T cells. J Exp Med 2000; 191: 551-560. 
7 Bhaskar S, Khanna SP, Mukherjee R. Isolation, purification and immunological characterization of novel low molecular weight protein antigen CFP 6 from culture filtrate of M. tuberculosis. Vaccine 2000; 18: 2856-2866.

8 Dhiman N, Verma I, Khuller GK. Cellular immune responses to cell wall peptidoglycan associated protein antigens in tuberculosis patients and healthy subjects. Microbiol Immunol 1997; 41: 495-502.

9 Schwander SK, Torres M, Sada E, et al. Enhanced responses to Mycobacterium tuberculosis antigens by human alveolar lymphocytes during active pulmonary tuberculosis. J Infect Dis 1998; 178: 1434-1445.

10 Schwander SK, Torres M, Carranza CC, et al. Pulmonary mononuclear cell responses to antigens of Mycobacterium tuberculosis in healthy household contacts of patients with active tuberculosis and healthy controls from the community. J Immunol 2000; 165: 1479-1485.

11 Sable SB, Kumar R, Kalra M, et al. Peripheral blood and pleural fluid mononuclear cell responses to low-molecularmass secretory polypeptides of Mycobacterium tuberculosis in human models of immunity to tuberculosis. Infect Immun 2005; 73: 3547-3558.

12 National Tuberculosis and Respiratory Disease Association. Diagnostic standard and classification of tuberculosis. New York, Nat Tuber Respir Dis Assoc, 1969.

13 Kamala T, Paramasivan CN, Herbert D, Venkatesan P, Prabhakar R. Isolation and identification of environmental mycobacteria in the Mycobacterium bovis BCG trial area of South India. Appl Environ Microbiol 1994; 60: 2180-2183.

14 Jindal SK, Behera D, Dhand R, Kashyap S, Malik SK. Flexible fiberoptic bronchoscopy in clinical practice - a review of 100 procedures. Indian J Chest Dis Allied Sci 1985; 27: 153-158.

15 Hirsch CS, Ellner JJ, Rusell DG, Rich EA. Complement receptor-mediated uptake and tumor necrosis factoralpha-mediated growth inhibition of Mycobacterium tuberculosis by human alveolar macrophages. J Immunol 1994; 152: 743-753.

16 Subrahmanyam D. Studies on the polyglycerophosphatides of Mycobacterium tuberculosis. Can J Biochem Physiol 1964; 42: 1195-1201.

17 Protein-protein BLAST. http://www.ncbi.nlm.nih.gov/ BLAST/Blast.cgi Date last updated: September 7, 2001. Date last accessed: July 30, 2003.

18 Sable SB, Verma I, Behera D, Khuller GK. Human immune recognition-based multicomponent subunit vaccines against tuberculosis. Eur Respir J 2005; 25: 902-910.

19 Green LC, Wagner DA, Glogowski J, Skippez PL, Wishnok JS, Tannenbaum SR. Analysis of nitrate, nitrite and $\left[{ }^{15} \mathrm{~N}\right]$ nitrate in biological fluids. Anal Biochem 1982; 126: 131-138.

20 Sable SB, Verma I, Khuller GK. Multicomponent antituberculous subunit vaccine based on immunodominant antigens of Mycobacterium tuberculosis. Vaccine 2005; 23: 41754184.

21 Phyu S, Tadesse A, Mustafa T, Tadesse S, Jonsson R, Bjune $G$. Diversity of lung and spleen immune responses in mice with slowly progressive primary tuberculosis. Scand J Immunol 2000; 51: 147-154.

22 Cooper AM, Callahan JE, Keen M, Belisle JT, Orme IM. Expression of memory immunity in the lung following reexposure to Mycobacterium tuberculosis. Tuber Lung Dis 1997; 78: 67-73.

23 Rich EA, Torres M, Sada E, Finegan CK, Hamilton BD, Toossi Z. Mycobacterium tuberculosis (MTB)-stimulated production of nitric oxide by human alveolar macrophages and relationship of nitric oxide production to growth inhibition of MTB. Tuber Lung Dis 1997; 78: 247-255.

24 Padgett EL, Pruett SB. Evaluation of nitrite production by human monocyte-derived macrophages. Biochem Biophys Res Commun 1992; 186: 775-781.

25 Weinberg JB, Misukonis MA, Shami PJ, et al. Human mononuclear phagocyte inducible nitric oxide synthase (iNOS): analysis of iNOS mRNA, iNOS protein, biopterin, and nitric oxide production by blood monocytes and peritoneal macrophages. Blood 1995; 86: 1184-1195.

26 Denis M. Tumor necrosis factor and gronulocyte macrophage-colony stimulating factor stimulate human macrophage to restrict growth of virulent Mycobacterium avium and to kill avirulent $M$. avium: killing effector mechanism depends on the generation of reactive nitrogen intermediates. J Leukoc Biol 1991; 49: 380-387.

27 Carpenter E, Fray L, Gormley E. Antigen-specific lymphocytes enhance nitric oxide production in Mycobacterium bovis BCG-infected bovine macrophages. Immunol Cell Biol 1998; 76: 363-368.

28 Saito S, Nakano M. Nitric oxide production by peritoneal macrophages of Mycobacterium bovis BCG-infected or noninfected mice: regulatory role of T-lymphocytes and cytokines. J Leukoc Biol 1996; 59: 908-915.

29 Horwitz MA, Lee BW, Dillon BJ, Harth G. Protective immunity against tuberculosis induced by vaccination with major extracellular proteins of Mycobacterium tuberculosis. Proc Natl Acad Sci USA 1995; 92: 1530-1534.

30 McShane H, Pathan AA, Sander CR, et al. Recombinant modified vaccinia virus Ankara expressing antigen 85A boosts BCG-primed and naturally acquired antimycobacterial immunity in humans. Nat Med 2004; 10: 1240-1244.

31 Horwitz MA, Harth G, Dillon BJ, Maslesa-Galic S. Enhancing the protective efficacy of Mycobacterium bovis BCG vaccination against tuberculosis by boosting with the Mycobacterium tuberculosis major secretory proteins. Infect Immun 2005; 73: 4676-4683.

32 Wang J, Thorson L, Stokes RW, et al. Single mucosal, but not parenteral, immunization with recombinant adenoviral-based vaccine provides potent protection from pulmonary tuberculosis. J Immunol 2004; 173: 6357-6365.

33 Giri PK, Verma I, Khuller GK. Enhanced immunoprotective potential of Mycobacterium tuberculosis Ag85 complex protein based vaccine against airway Mycobacterium tuberculosis challenge following intranasal administration. FEMS Immunol Med Microbiol 2006; 47: 233-241.

34 Doherty TM. Real world TB vaccines: clinical trials in TBendemic regions. Vaccine 2005; 23: 2109-2114. 